\title{
Transonic Small-Disturbance Theory for Lightly Loaded Cascades
}

\author{
A. F. Messiter* and T. C. Adamson Jr. $\dagger$ \\ University of Michigan, Ann Arbor, Mich.
}

\begin{abstract}
Analytical solutions are derived for a class of two-dimensional transonic cascade flows. In the particular limiting case studied, the airfoils are considered as lightly loaded; a similarity parameter is defined for this case. Second-order asymptotic solutions are derived for the nearly one-dimensional flow in regions directly between successive airfoils and for the periodic flow ahead of and behind the cascade. For high subsonic Mach numbers, composite solutions are obtained by a suitable joining of these results with solutions derived for thin regions containing the leading and trailing edges. Surface pressures and lines of constant Mach number are calculated for circular-arc airfoils in some specific examples. For low supersonic speeds a far-field solution, which allows calculation of the decay of shock waves at large distances, is also derived.
\end{abstract}

\section{Introduction}

$\mathbf{S}$ UCCESSFUL calculation of three-dimensional transonic flows in turbomachinery depends in part on understanding the two-dimensional flow through a stationary cascade of airfoils. Important physical features of the threedimensional flows are preserved in the plane case, and solutions for two-dimensional flows may have direct application in three-dimensional solutions. Even for twodimensional flows a wide variety of possibilities arises, in terms of both blade geometry and flow structure. Comprehensive discussions of the two-and three-dimensional flow problems have appeared, e.g., in Refs. 1-3. Analytical studies, however, have not yet taken full advantage of the simplifications that might be achieved, at least in certain cases, by systematic expansions in terms of suitable small parameters.

Transonic flow theory has usually focused on either external or internal flows, but seldom on a combination of the two. The transonic small-disturbance approximation, derived in the first step of an asymptotic expansion procedure involving two or more small parameters, ${ }^{4}$ has been widely used for the description of flow past thin wings and bodies at Mach numbers near one. Second approximations in a number of different cases have also been studied. ${ }^{5}$ A different type of transonic flow occurs in channels and nozzles and can be described by relatively simple solutions. ${ }^{6}$ Here the first term in an asymptotic flow description is typically a onedimensional approximation, if the area changes are gradual and can therefore be characterized by a small parameter. The nonlinear transonic small-disturbance equation is needed only in certain particular cases where streamwise gradients are locally large ${ }^{7}$; in some situations a local two-dimensional approximation is correctly given by linear equations. ${ }^{8}$ Asymptotic solutions including higher-order terms and local solutions have been derived for a variety of steady and unsteady flows. ${ }^{8-11}$

The possibility of extending these asymptotic flow descriptions to include flows through a cascade of airfoils appears still unexplored. It is evident, however, that simplifications can be achieved because the blade spacing is typically comparable with the chord length, whereas the proper transverse length scale (in the direction normal to streamlines) according to transonic small-disturbance theory is large. In the two-dimensional case the implication is, as will

Received Oct. 14, 1980; revision received March 6, 1981. Copyright (C) by A. F. Messiter and T. C. Adamson Jr. Published by the American Institute of Aeronautics and Astronautics with permission.

*Professor of Aerospace Engineering. Member AIAA.

$\dagger$ Professor of Aerospace Engineering. Associate Fellow AIAA. be shown later, that a portion of the flow directly between any two adjacent airfoils is described in a first approximation as a one-dimensional (internal). flow, whereas the flow outside these regions, expressed in terms of the larger length scale, resembles the (external) flow past a nearly plane wall with a certain periodic shape. In the channel part of the flow the important small geometric parameter measures a relative area change and so depends on thickness and camber. Various limiting cases can be studied, depending on the relative sizes of the area-change parameter, the angle of attack, and the difference between the undisturbed Mach number and one. In one special case, when the two geometric parameters are of the same order, the flow ahead of the channel region is purely subsonic or supersonic and the equations are found to be linear (except for the far-field in the supersonic case); thus, the airfoils would be considered lightly loaded. In another particular case, when the angle of attack is somewhat larger and the airfoils are therefore more heavily loaded, the nonlinear transonic small-disturbance equation is required for the first approximation. In either case it would be found that solutions in thin leading- and trailing-edge regions are also needed. The advantage of these formulations, of course, is that the overall flow problem can be divided into a number of simpler problems, solutions to which can, at least in principle, be carried out to higher order. The results then are assembled in the form of composite solutions for numerical evaluation in specific examples.

The present work is concerned with lightly loaded airfoils, such that the angle of attack is of the same order as the parameter which measures thickness and camber. Some general features of this case are outlined in Sec. II. First- and second-order solutions for subsonic flow are shown in Sec. III, with surface pressures and lines of constant Mach number plotted for a few numerical examples. For supersonic flow, solutions are discussed in Sec. IV for the flow ahead of the cascade, including the far field. Only the main steps in the analyses are shown here; additional details are given in Ref. 12 , available from the authors.

\section{General Features}

Inviscid flow at transonic speeds through a twodimensional cascade of thin airfoils is characterized by the values of the Mach number and of various nondimensional geometric parameters (Fig. 1). In the present case the thickness ratio, camber, and angle of attack $\alpha$ of the airfoils are all taken to be of the same small order of magnitude. For $\alpha=0$ the upper and lower surfaces, measured relative to the chord line, are denoted by $y=\epsilon^{2} f_{u}(x)$ and $y=\epsilon^{2} f_{\ell}(x)$, respectively, where $\epsilon^{2} \ll 1$ and $x$ and $y$ are rectangular coor- 


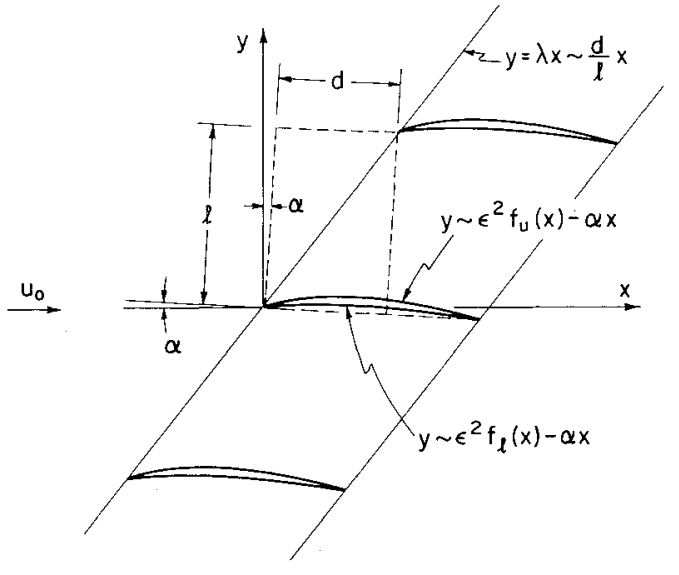

Fig. 1 Flow geometry.

dinates made nondimensional with the chord length; the origin is at the leading edge of one of the airfoils. For $\alpha \neq 0, x$ is measured in the direction of the undisturbed flow; since $|\alpha| \ll 1$, the surfaces are given approximately by $y \sim \epsilon^{2} f_{u, q}$ $-\alpha x$. The airfoil spacing $\ell$ (measured normal to the chord line), also nondimensional with the chord, and the stagger angle $\tan ^{-1}(d / \ell)$ are of order one. The straight line passing through the leading edges is denoted by $y=\lambda x$, where $\lambda=\tan \left\{\tan ^{-1}(d / \ell)+\alpha\right\}=(d / \ell)+O(\alpha)$. Velocity components $u$ and $v$, nondimensional with the sound speed in the undisturbed flow, are in the $x$ and $y$ directions, respectively. Thermodynamic quantities are nondimensional with their values in the undisturbed flow.

At large distances ahead of the cascade, for $y-\lambda x \gg 1$, the Mach number $M_{0}=u_{0}$ is close to one. Various approximation procedures might be considered, depending on the relative sizes of $M_{D}^{2}-1$ and $\epsilon$. For example, transonic smalldisturbance theory for a single airfoil is derived for $M_{0}^{2}$ $-1=O\left(\epsilon^{4 / 3}\right)$. This ordering has sometimes also been suggested for a cascade (e.g., by Oswatitsch ${ }^{3}$ ). As explained below, the choice made here is instead

$$
M_{0}^{2}-1= \pm K \epsilon
$$

where $K$ is a positive constant and the upper and lower signs refer to the supersonic and subsonic cases, respectively. Then also

$$
M_{0}=u_{0}=1 \pm \epsilon k(\epsilon)
$$

where $k(\epsilon)$ is simply related to $K$ by $K=2 k \pm k^{2} \epsilon$. Results of linear theory for flow past a single airfoil, and therefore also of transonic small-disturbance theory when the usual similarity parameter is large, indicate that a differential equation having solutions capable of satisfying boundary conditions both at the airfoil and at infinity is obtained for $\epsilon \rightarrow 0$ in terms of coordinates $x$ and $\epsilon^{1 / 2} y$. The velocity and pressure perturbations are then found to be $O\left(\epsilon^{3 / 2}\right)$, small in comparison with $\left|M_{0}^{2}-1\right|$. However, in the present case the airfoil spacing is $O(1)$ rather than $O\left(\epsilon^{-1 / 2}\right)$, and in the region between two adjacent airfoils the changes in the $y$ direction are therefore small, so that the fluid motion is nearly onedimensional, just as in a channel flow. If the distance between airfoil surfaces is $\ell+O\left(\epsilon^{2}\right)$, for an entrance Mach number $1+O(\epsilon)$, the pressure and velocity changes in this channel-like flow are found to be $O(\epsilon)$, larger than the $O\left(\epsilon^{3 / 2}\right)$ changes for an isolated airfoil. If the width $\ell+\epsilon^{2} f_{\ell}(x-d)-\epsilon^{2} f_{u}(x)$ has a minimum for $d<x<1$, the flow will become choked at a sufficiently small value of $K$. The constant $K$ might be regarded as a similarity parameter for cascades, analogous to the usual transonic similarity parameter for a single airfoil: if $\left(M_{0}^{2}-1\right) / \epsilon= \pm K$ is held constant as $\epsilon \rightarrow 0$, the largest changes in local Mach number are of the same order as the difference between the undisturbed-flow Mach number and one. It is for this reason that the relation given by $\mathrm{Eq}$. (1) represents an important special case.

Far ahead of the cascade, the normal distance between two streamlines which later intersect the leading edges of adjacent airfoils is $\ell-\alpha d+O\left(\alpha^{2}\right)$. The nondimensional mass flow between two such streamlines is $u_{0}(\ell-\alpha d)+O\left(\alpha^{2}\right)$. At the entrance to the region directly between adjacent airfoils the distance between the same two streamlines is $\ell-\epsilon^{2} f_{u}(d)+\ldots$. If the flow entering this region has a velocity which differs from the undisturbed value $u_{0}=1 \pm k(\epsilon) \epsilon$ only by terms of higher order than $\epsilon$, the mass flow between the blades is found to be $\left(u_{0}+\ldots\right) \cdot\left(\ell-\epsilon^{2} f_{u}(d)+\ldots\right)$, in agreement with the first result only if $\alpha=\epsilon^{2} f_{u}(d) / d+\ldots$ As pointed out in the following sections, this value is the first approximation to the "unique incidence angle" at supersonic speeds (see Ref. 1, Sec. G; Refs. 13 and 14) and to the angle for zero flow around the leading edge at subsonic speeds.

The velocity components must satisfy the differential equation

$$
a^{2} \operatorname{div} q=q \cdot \nabla\left(q^{2} / 2\right)
$$

where $q, q$, and $a$ are, respectively, the velocity vector, the magnitude of the velocity, and the local sound speed, all nondimensional with the sound speed in the undisturbed flow. For a perfect gas with uniform total enthalpy,

$$
a^{2}=1+1 / 2(\gamma-1)\left(u_{0}^{2}-q^{2}\right)
$$

where $\gamma$ is the ratio of specific heats. For subsonic speeds the flow is irrotational, and a perturbation potential $\phi$ can be defined by

$$
u=u_{0}+\phi_{x} \quad v=\phi_{y}
$$

For supersonic speeds the introduction of a potential remains a correct approximation to the order required here, since the vorticity associated with entropy gradients behind shock waves is of sufficiently high order as $\epsilon \rightarrow 0$. The largest terms in the differential equation then give

$$
\begin{aligned}
& \phi_{y y}=\left\{ \pm K \epsilon+(\gamma+1) u_{0} \phi_{x}\right\} \phi_{x x}+(\gamma-1) \phi_{x} \phi_{y y} \\
& \quad+2 \phi_{y} \phi_{x y}+1 / 2(\gamma+1) \phi_{x}^{2} \phi_{x x}+\cdots
\end{aligned}
$$

A tangency condition is to be satisfied at each of the airfoil surfaces:

$$
\begin{aligned}
& \phi_{y}\left(x, n \ell^{+}\right)=\epsilon^{2} f_{u}^{\prime}(x-n d)-\alpha+\cdots \\
& \phi_{y}\left(x, n \ell^{-}\right)=\epsilon^{2} f_{\ell}^{\prime}(x-n d)-\alpha+\cdots
\end{aligned}
$$

for $0<x-n d<1+\ldots$ and $n=0, \pm 1, \pm 2, \ldots$ For $M_{0}<1$, the flow is uniform far ahead of and behind the cascade and a Kutta condition is used at the trailing edges. For $M_{0}>1$, no disturbances originate ahead of the cascade.

As already anticipated, in a "channel" region between two adjacent airfoils the length $l-d$ and width $\ell$ are of the same order, and the solution will be expressed in terms of coordinates $x$ and $y$. Ahead of and behind the cascade, however, the solutions obtained in terms of these variables, which might be called "near-field" solutions, do not satisfy the boundary conditions at large distances. The proper transverse length scale is larger, so that here $x=O(1)$ and $y=O\left(\epsilon^{-1 / 2}\right)$; the solution will be periodic in $x$ for constant values of a coordinate $\epsilon^{1 / 2}(y-\lambda x)$. To this scale the distance $\epsilon^{1 / 2} \ell$ between airfoils disappears in the limit as $\epsilon \rightarrow 0$. The "outer" flow ahead of the cascade is then found to be the same, in a first approximation, as the flow past a "wall" having a periodic scalloped shape such as sketched in Fig. 2a; the downstream flow is described in a similar way. In addition, it will be seen 


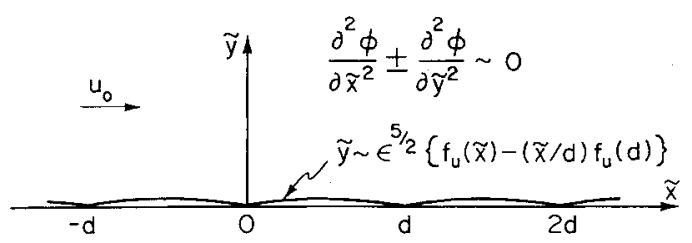

a) Approximate flow problem at large distances.

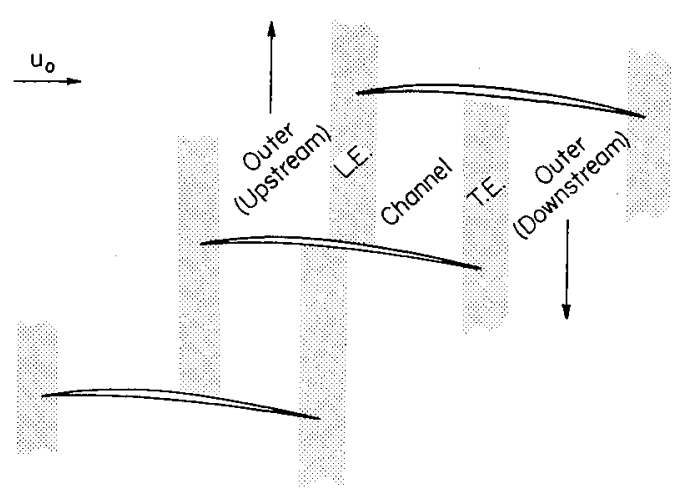

b) Outer, edge, and channel flow regions.

Fig. 2 Asymptotic flow description.

that a direct asymptotic matching of "outer" and "channel" solutions is not possible and that "edge" solutions in thin vertical strips containing the leading and trailing edges are also needed. Again following small-disturbance theory, one would anticipate that the width of these "edge" regions is smaller than the height by a factor $O\left(\epsilon^{1 / 2}\right)$, and therefore that the solutions here are to be found in the limit as $\epsilon \rightarrow 0$ with coordinates $(x-n d) / \epsilon^{1 / 2}$ and $(y-n \ell)$ held fixed, for $n=0, \pm 1$, $\pm 2, \ldots$. These regions are indicated by the shaded areas in Fig. 2b. Finally, for supersonic speeds the cumulative effect of small errors in the linear theory for flow past a single airfoil can no longer be neglected ${ }^{15}$ when $y=O\left\{\left(M_{0}^{2}-1\right) / \epsilon^{2}\right\}$, if streamline slopes are $O\left(\epsilon^{2}\right)$; in the present case, for $M_{0}^{2}$ $1=O(\epsilon)$, a "far-field" description is needed for $y=O\left(\epsilon^{-I}\right)$.

\section{Subsonic Flows}

Suitable forms for the asymptotic expansions of the perturbation potential are determined in a stepwise fashion by use of the differential equation (6) and the boundary and matching conditions for the outer, edge, and channel regions. The derivations are fairly straightforward but tedious, and only the results are shown here. Additional details are given in Ref. 12. Terms of order $\epsilon^{2}$ in $u$ are obtained for the outer and channel regions, but only terms of order $\epsilon^{3 / 2}$ are shown for the edge regions. These latter regions are small and the higherorder solutions are complicated; the possible improvement in accuracy does not seem great enough to justify including the higher-order calculations.

In the limit as $\epsilon \rightarrow 0$ with $x$ and $y$ held fixed, the first-order approximation to the exact potential equation becomes simply $\phi_{y y}=0$. For $d<x<1$ and $0<y<\ell$, in the channel region, it is convenient to write the expansion of $\phi$ in the form

$$
\phi=\left(a^{*}-u_{0}\right) x+\epsilon \phi_{I}(x, y ; \epsilon)+\epsilon^{2} \phi_{2}(x, y ; \epsilon)+\cdots
$$

where $\phi_{I}, \phi_{2}, \ldots$ are bounded as $\epsilon \rightarrow 0$, and certain simplifications in higher-order terms are achieved by the incorporation of fractional powers and logarithms of $\epsilon$ in the functions $\phi_{1}, \phi_{2}, \ldots$. The solutions for $u$ and $v$ which satisfy the tangency conditions at $y=0$ and $y=\ell$ are

$$
u=a^{*}+\epsilon \phi_{I}^{\prime}(x ; \epsilon)+\epsilon^{2} \phi_{2 x}(x, y)+\cdots
$$

$$
v=-\alpha+\epsilon^{2}\left\{\left(1-\frac{y}{\ell}\right) f_{u}^{\prime}(x)+\frac{y}{\ell} f_{\ell}^{\prime}(x-d)\right\}+\cdots
$$

where

$$
\begin{aligned}
& \phi_{I}^{\prime 2}(x)=c_{I}^{2}(\epsilon)-\frac{2 a^{* 2}}{(\gamma+1) \ell}\left\{f_{u}(x)-f_{u}(d)-f_{\ell}(x-d)\right\} \\
& \phi_{2 x}(x, y)=\frac{\ell}{2}\left(\frac{y^{2}}{\ell^{2}}-\frac{l}{3}\right) f_{\ell}^{\prime \prime}(x-d) \\
& -\frac{\ell}{2}\left(\frac{(y-\ell)^{2}}{\ell^{2}}-\frac{l}{3}\right) f_{u}^{\prime \prime}(x)+\frac{3-2 \gamma}{6} \phi_{1}^{2} \\
& \left(\epsilon c_{l}\right)^{2}=\left(a^{*}-u_{0}\right)^{2}\left\{1+2 \frac{(\gamma+1) d}{K^{2} \ell}\left(\frac{\alpha}{\epsilon^{2}}-\frac{f_{u}(d)}{d}\right)\right. \\
& \left.+\frac{(3-2 \gamma) K \epsilon}{3(\gamma+1)}\right\}+\cdots
\end{aligned}
$$

and $a^{* 2}=1-(\gamma-1) K \epsilon /(\gamma+1)$; the lower sign is taken in the definition of $K$ from Eq. (1). The results for $\phi_{1}$ and $\phi_{2}$ are identical to those for a two-dimensional channel. ${ }^{8}$ The functions of $x$ in $\phi_{1}$ and $\phi_{2}$ are found, respectively, by satisfying the boundary conditions for $\phi_{2 y}$ and $\phi_{3 y}$. The result for $c_{l}$ is found by balancing the mass flow as $x-d \rightarrow 0$ with the mass flow at a large distance ahead of the cascade. As noted in the preceding section, the angle of attack $\alpha$ is taken to be $O\left(\epsilon^{2}\right)$. It is anticipated here that $\alpha$ should be expanded in terms of $\epsilon$. The first term is chosen to have the specific value discussed previously, and it is seen later that the second term is typically $O\left(\epsilon^{5 / 2}\right)$ :

$$
\alpha=\epsilon^{2} f_{u}(d) / d+\epsilon^{5 / 2} \alpha_{5 / 2}+\cdots
$$

The value of the coefficient $\alpha_{5 / 2}$ is considered to be known. In Eq. (13), the bracket then contains a term of order $\epsilon^{1 / 2}$; this term can also be found from the second-order outer solution derived below.

Ahead of this region, for $0<x<d$ and $0<y<\infty$, the streamlines are not constrained by a second boundary surface as in a channel. Changes in the streamtube area are not as large, and the flow is in some respects similar to the flow over a single airfoil. Pressure changes are $O\left(\epsilon^{3 / 2}\right)$ rather than $O(\epsilon)$, and so the changes in local Mach number are small in comparison with $\left|M_{0}-1\right|$. The "near-field" solution for $0<x<d$ and $y=O(1)$ has the form

$$
\begin{gathered}
u=u_{0}+\epsilon^{3 / 2} \phi_{3 / 2}^{\prime}(x)+\epsilon^{2}\left\{y f_{u}^{\prime \prime}(x)+h_{2}^{\prime}(x)\right\}+\cdots \\
v=\epsilon^{2}\left\{f_{u}^{\prime}(x)-f_{u}(d) / d\right\}+\epsilon^{5 / 2}\left\{-K y \phi_{3 / 2}^{\prime \prime}(x)-\alpha_{5 / 2}\right\}+\cdots
\end{gathered}
$$

where $\phi_{3 / 2}^{\prime}(x)$ and $h_{2}^{\prime}(x)$ are to be determined by matching with the solution obtained for larger distances.

For $0<x<d$, a convenient representation of the upper airfoil surface shows quadratic and cubic parts separately, with the remaining shape details expressed by a Fourier series having period $d$ :

$$
\begin{gathered}
f_{u}(x)=\frac{x}{d} f_{u}(d)-\frac{1}{2} \Delta_{u} d\left(\frac{x}{d}-\frac{x^{2}}{d^{2}}\right) \\
+\frac{1}{12} \delta_{u} d^{2}\left(\frac{x}{d}-\frac{3 x^{2}}{d^{2}}+\frac{2 x^{3}}{d^{3}}\right)+g_{u}(x)
\end{gathered}
$$

Here the differences in slope and in curvature at $x=0$ and $x=d$ are shown explicitly by $\Delta_{u}=f_{u}^{\prime}(d)-f_{u}^{\prime}(0)$ and $\delta_{u}=f_{u}^{\prime \prime}(d)-f_{u}^{\prime \prime}(0)$, and

$$
g_{u}(x)=\sum_{n=1}^{\infty}\left(a_{n} \cos \frac{2 n \pi x}{d}+b_{n} \sin \frac{2 n \pi x}{d}\right)
$$


If $f_{u}^{\prime \prime}(x)$ is continuous for $0<x<d$, and since $g_{u}^{\prime \prime}(0)=g_{u}^{\prime \prime}(d)$, it follows that $a_{n}=O\left(1 / n^{4}\right)$ and $b_{n}=O\left(1 / n^{4}\right)$ as $n \rightarrow \infty$. This representation assumes a sharp leading edge. For a local rounding of the leading edges, the airfoil shape [Eq. (17)] can be modified by a simple additive correction, which then requires a source term proportional to $\ln z^{*}$ in the leading-edge solution of Eq. (24), as well as corresponding small corrections in $\alpha$ and in the "channel" width in Eq. (11). ${ }^{12}$

The outer solution is expressed in terms of coordinates $\tilde{x}$ and $\tilde{y}$ defined by

$$
\tilde{x}=x, \quad \tilde{y}=\epsilon^{1 / 2} K^{1 / 2}(y-\lambda x)
$$

Along lines $\bar{y}=$ const the flow perturbations are periodic functions of $\tilde{x}$ with period $d$. The potential has the form

$$
\phi=\epsilon^{3 / 2} \tilde{\phi}_{3 / 2}(\tilde{x}, \tilde{y})+\epsilon^{2} \tilde{\phi}_{2}(\tilde{x}, \tilde{y})+\cdots
$$

and it is found that $\tilde{\phi}_{3 / 2}$ satisfies Laplace's equation. In the solution for the complex velocity, the term of order $\epsilon^{2}$ which decreases exponentially as $\bar{y} \rightarrow \infty$ and matches correctly with the solution for $y=O(1)$ is, with $\tilde{z}=\tilde{x}+i \tilde{y}$,

$$
\begin{aligned}
& K^{1 / 2}\left(\tilde{\phi}_{(3 / 2) \tilde{x}}-i \tilde{\phi}_{(3 / 2) \bar{y}}\right)=-\frac{\Delta_{u}}{\pi} \ln \left(1-e^{2 \pi i z / d}\right)-\frac{i}{12} \delta_{u} d \\
& -\frac{1}{\pi} \delta_{u} \int_{0}^{\tilde{z}} \ln \left(1-e^{2 \pi i z / d}\right) \mathrm{d} \tilde{z}+\frac{2 \pi}{d} \sum_{n=1}^{\infty} n\left(a_{n}-i b_{n}\right) e^{i 2 n \pi \tilde{z} / d}
\end{aligned}
$$

This is the same as the solution for the flow past the periodic wall shape sketched in Fig. 2a. The matching also gives

$$
\phi_{3 / 2}(x)=\tilde{\phi}_{3 / 2}(x, 0)
$$

The "outer" solution for $0<x<d$ cannot be matched directly as $\tilde{z} \rightarrow d$ with the "channel" solution for $d<x<1$. The logarithmic singularity in the velocity as $\tilde{z} \rightarrow n d$ indicates the need for inner solutions in narrow vertical strips, each containing one of the leading edges (Fig. 2b). Coordinates $x^{*}$ and $y^{*}$ are defined by

$$
x^{*}=\frac{\pi(x-n d)}{\epsilon^{1 / 2} K^{1 / 2} \ell} \quad y^{*}=\frac{\pi(y-n \ell)}{\ell}
$$

for $n=0, \pm 1, \pm 2, \ldots$ In the limit as $\epsilon \rightarrow 0$ with $x^{*}$ and $y^{*}$ fixed, the flow is studied in the half-plane $y^{*}>-\pi$ with a semi-infinite slit $y^{*}=0, x^{*}>0$. The potential can be written in the form

$$
\phi=\epsilon^{2}(\ell / \pi) \phi_{2}^{*}\left(x^{*}, y^{*} ; \epsilon\right)+\cdots
$$

where $\phi_{2}^{*}$ is found to satisfy Laplace's equation, and the matching with the outer solution implies $\phi_{2}^{*}=O(\ln \epsilon)$ as $\epsilon \rightarrow 0$. A conformal transformation

$$
z^{*}=\zeta-1-\ln \zeta
$$

where $z^{*}=x^{*}+i y^{*}$, and $\zeta=\xi+i \eta$ maps the flow onto the upper half of the $\zeta$ plane. The solution for the complex perturbation velocity is

$$
\begin{array}{r}
\phi_{2 x^{*}}^{*}-i \phi_{2 y^{*}}^{*}=-i f_{u}^{\prime}(0)+\frac{l}{\pi} \Delta_{\ell e} \ln (\zeta-1) \\
-\frac{1}{\pi} \Delta_{d} \ln \zeta+i f_{u}(d) / d+c_{2}^{*}(\epsilon)+\frac{m_{2} / \ell}{\zeta-1}
\end{array}
$$

where $\Delta_{\ell e}=f_{u}^{\prime}(0)-f_{\ell}^{\prime}(0), \Delta_{d}=f_{u}^{\prime}(d)-f_{\ell}^{\prime}(0)$, and $c_{2}^{*}$ and $m_{2}$ are real constants to be determined. Matching as $\zeta \rightarrow \infty$ with the outer solution evaluated as $\tilde{z} \rightarrow n d$ gives

$$
c_{2}^{*}=\frac{\Delta_{u}}{2 \pi} \ln \frac{d^{2}}{4 \epsilon K \ell^{2}}+\frac{2 \pi}{d} \sum_{n=1}^{\infty} n a_{n}
$$

A positive or negative value of $m_{2}$ corresponds, respectively, to an upward or downward flow around the leading edge, with complex perturbation velocity locally proportional to $\left(z^{*}\right)^{-1 / 2}$. Matching as $\zeta \rightarrow 0$ with the solution of Eq. (9) evaluated for $x \rightarrow d$ gives a relation between $c_{1}(\epsilon)$ and $m_{2}$, or between $m_{2}$ and $\alpha_{5 / 2}$ if Eq. (13) for $c_{1}$ is used:

$$
\frac{m_{2}}{\ell}=\frac{d}{\ell K^{1 / 2}} \alpha_{5 / 2}+\frac{\Delta_{d}}{\pi}+c_{2}^{*}
$$

The edge solution of Eq. (26) contains terms of order $1 / z^{*}$ as $\zeta \rightarrow \infty$, and so the complex velocity in the outer solution must have terms $O\left\{\epsilon^{2} /(\tilde{z}-n d)\right\}$ as $\tilde{z} \rightarrow n d$, for $n=0, \pm 1, \pm 2$, .... That is, point sources at $\tilde{z}=n d$ must be included in $\tilde{\phi}_{2}$. In the case considered here, for $\alpha=\epsilon^{2} f_{u}(d) / d+\ldots$, such source terms are excluded from $\tilde{\phi}_{3 / 2}$. The perturbation velocity in the leading-edge region is therefore $O\left(\epsilon^{3 / 2}\right)$ for this case, rather than $O(\epsilon)$, and so the nonlinear transonic small-disturbance equation is not required. Moreover, there is no change of order $\epsilon^{2}$ in the mass flow in the edge region; a change of this order would imply a different numerical coefficient for the first term in $\alpha$. It follows also that the value $\alpha=\epsilon^{2} f_{u}(d) / d$ is the first term of the expansion as $\epsilon \rightarrow 0$ for the particular angle of attack at which there is no flow around the leading edge; the second term is the value found for $\epsilon^{5 / 2} \alpha_{5 / 2}$ when $m_{2}=0$.

The term $\epsilon^{2} \tilde{\phi}_{2}$ in the outer solution can be represented in three parts. There is a singular part $\widetilde{\phi}_{2}^{(s)}$ which has source-like behavior at $\tilde{z}=n d$ and which is, for convenience, also required to satisfy the boundary condition at large distances. A particular solution $\tilde{\phi}_{2}^{(p)}$ appears because the differential equation for $\tilde{\phi}_{2}$ is nonhomogeneous. Finally, an additional solution $\tilde{\phi}_{2}^{(h)}$ to the homogeneous equation is necessary because a simple choice for $\tilde{\phi}_{2}^{(p)}$ does not satisfy the required matching condition as $\tilde{y} \rightarrow 0 .{ }^{12}$ One finds also that the expansion of $\epsilon^{3 / 2} \tilde{\phi}_{3 / 2}(\tilde{x}, \tilde{y})$ for small $\tilde{y}$, rewritten in terms of $x$ and $y$, leads to terms of order $\epsilon^{2}$ which contribute to the matching. ${ }^{12}$ The results for the three parts of $\tilde{\phi}_{2}(\tilde{x}, \tilde{y})$ are given by

$$
\begin{gathered}
\tilde{\phi}_{2 \tilde{x}^{(s)}}-i \tilde{\phi}_{2 \tilde{y}}^{(s)}=\left\{-\frac{\Delta_{u}}{\pi} \ln \left(1-e^{2 \pi i \tilde{z} / d}\right)-\frac{\Delta_{u}}{2 \pi} \ln \frac{d^{2}}{4 \epsilon K \ell^{2}}\right. \\
\left.+\frac{m_{2}}{\ell}-\frac{\Delta_{d}}{\pi}-\frac{\Delta_{u}}{2} i\right\} \frac{\ell}{d} \operatorname{ctn} \frac{\pi \tilde{z}}{d} \\
\tilde{\phi}_{2}^{(p)}=K^{1 / 2} \frac{\ell}{d} \tilde{y} \tilde{\phi}_{(3 / 2) \tilde{x}}+\frac{\gamma+1}{4 K}\left(\tilde{\phi}_{3 / 2} \tilde{\phi}_{(3 / 2) \tilde{x}}-\tilde{y} \tilde{\phi}_{(3 / 2) \tilde{x}} \tilde{\phi}_{(3 / 2) \tilde{y}}\right) \\
\tilde{\phi}_{2 \bar{x}^{(h)}}-i \tilde{\phi}_{2 \tilde{y}}^{(h)}=\frac{\ell \Delta_{u}}{2 d}+i \frac{\alpha_{5 / 2}}{K^{1 / 2}}+i \frac{\ell}{d} K^{1 / 2}\left(\tilde{\phi}_{(3 / 2) \bar{x}}-i \tilde{\phi}_{(3 / 2) \tilde{y}}\right) \\
+i \frac{(\gamma+1)}{4 K^{3 / 2}} \frac{\Delta_{u}}{d}\left(\tilde{\phi}_{3 / 2}-A_{0}+i \tilde{\psi}_{3 / 2}\right)+F(\tilde{x}, \tilde{y})-i G(\tilde{x}, \tilde{y})
\end{gathered}
$$

The integration constant in $\tilde{\phi}_{3 / 2}$ is chosen so that $\tilde{\phi}_{3 / 2}(\tilde{x}, 0)=0$ at $\tilde{x}=n d$, to eliminate source-like behavior in $\tilde{\phi}_{2}^{(p)}$ at these points; additional constants are chosen such that the complex potential $\tilde{\phi}_{3 / 2}-A_{0}+i \widetilde{\psi}_{3 / 2} \rightarrow 0$ as $\tilde{y} \rightarrow \infty$. The term $F-i G$ is zero for a circular-arc airfoil and is therefore not needed in the numerical examples described below; the determination of $F-i G$ for more general airfoil shapes is discussed in Ref. 12. Further calculations show that the requirement $\tilde{\phi}_{2 y} \rightarrow 0$ as $\tilde{y} \rightarrow \infty$ leads again to the result in Eq. (28) relating the source strength $\epsilon^{2} m_{2}$ to the perturbation $\epsilon^{5 / 2} \alpha_{5 / 2}$ in the angle of 


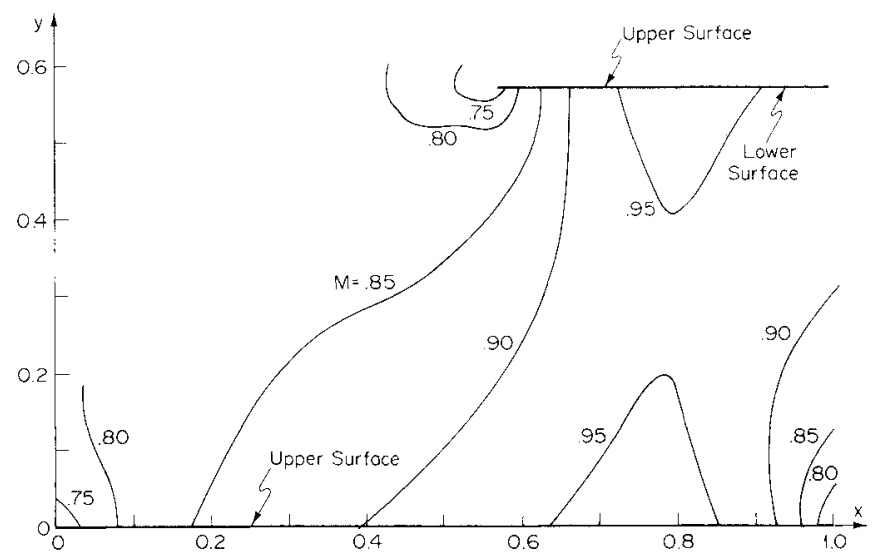

a) $y_{c}=0, \alpha=1.0 \mathrm{deg}$.

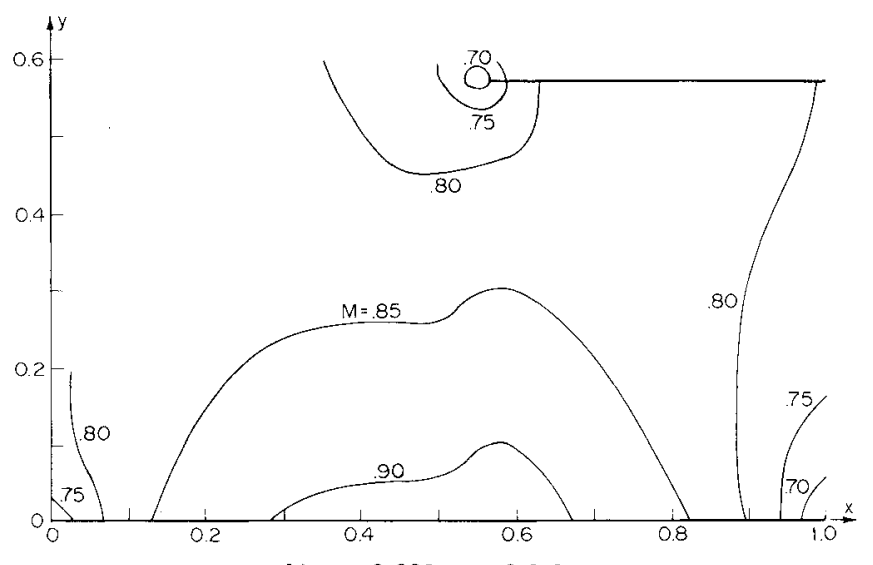

b) $y_{c}=0.009, \alpha=2.2 \mathrm{deg}$.

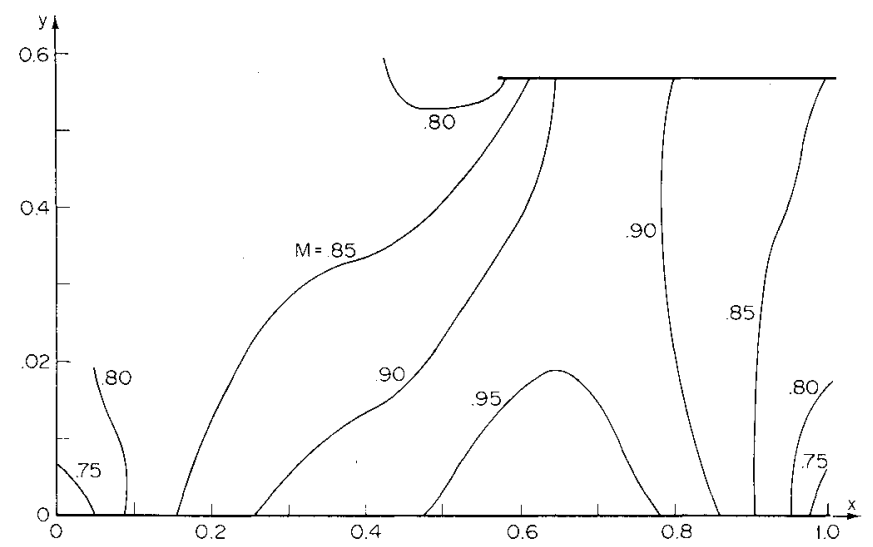

c) $y_{c}=0.009, \alpha=1.3 \mathrm{deg}$.

Fig. 3 Lines of constant Mach number for circular-arc profiles with thickness ratio $t / c=\epsilon^{2}\left(\Delta_{\ell}^{-} \Delta_{\mu}\right) / 8=0.035$, stagger angle $\tan ^{-1}(d / \ell)$ $=45 \mathrm{deg}$, solidity $\left(\ell^{2}+d^{2}\right)-1 / 2=1.25$, and Mach number $M_{0}=0.815$ (values of camber $y_{c}=-\epsilon^{2}\left(\Delta_{u}+\Delta_{\ell}\right) / 16$ and angle of attack $\alpha$ are varied).

attack. It follows that the term of order $\epsilon^{1 / 2}$ in $c_{l}$, obtained previously from overall mass-flow considerations, could instead have been found from the solution for $\tilde{\phi}_{2}$.

Three composite solutions have been constructed for use in numerical calculations. For $0<y<\ell$ and $d<x<1$, leadingand trailing-edge solutions for $u_{0}+\phi_{x}$, where $\phi$ has the form of Eq. (24), are added to the channel solution [Eq. (9)] and the common parts are then subtracted. The Kutta condition is satisfied in the trailing-edge solution by omission of a source term analogous to the term $\left(m_{2} / t\right) /(\zeta-1)$ which appears in Eq. (26); the presence of such a term would imply flow around the edge. For $0<y<\lambda x$ and $0<x<d$, an additive composition is again used to combine the near-field solution [Eq. (15)] with leading-edge solutions about $x=0$ and $x=d$.

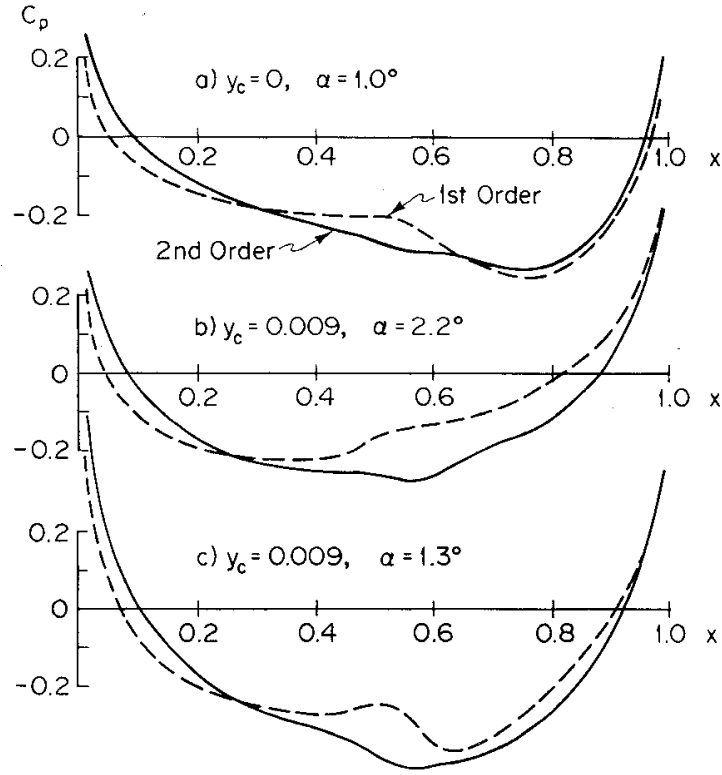

Fig. 4 Pressure coefficient at upper airfoil surface (suction surface) for the same three cases as in Fig. 3 (dashed and solid lines denote first and second approximations, respectively).

For $y>\lambda x$ and $0<x<d$, the outer solution [Eq. (20)] and the edge solutions are combined in the same way. Here the nearfield solution consists simply of the first terms in an expansion of the outer solution for small $\tilde{y}$. The outer solution is retained, however, to avoid the expansion of $\exp (-\pi \tilde{y} / d)$ for small $\tilde{y}$, which quickly loses accuracy as $y-\lambda x$ increases. These composite solutions contain terms of order $\epsilon^{2}$ in the velocity perturbations everywhere except in the edge regions, where only terms of order $\epsilon^{3 / 2}$ are retained. At $x=d$ a simple interpolation formula is used to join the terms of order $\epsilon^{2}$ obtained from the channel and near-field solutions, and simple smoothing functions are introduced to eliminate the discontinuities in higher-order terms. ${ }^{12}$

Lines of constant Mach number and pressures on an upper blade surface are plotted in Figs. 3 and 4 for three examples with circular-arc blades. For these profiles, the flow details depend on six parameters: spacing, stagger, thickness, camber, angle of attack, and Mach number. In the examples chosen, the Mach number and the first three geometric parameters are fixed, while the camber and angle of attack are varied. The blade spacing measured along the straight line through the leading edges is taken to be $80 \%$ of the chord, so that $\left(\ell^{2}+d^{2}\right)^{1 / 2}=0.8$; this is the reciprocal of the solidity. The stagger angle $\tan ^{-1}(d / \ell)$ is $45 \mathrm{deg}$. Upper and lower surfaces, measured relative to the chord line, are defined by $\epsilon^{2} f_{u, \ell}(x)=-1 / 2 \epsilon^{2} \Delta_{u, \ell}\left(x-x^{2}\right) / d$; the thickness ratio $t / c=1 / 8\left(\Delta_{\ell}-\Delta_{u}\right) \epsilon^{2} / d$ is taken to be 0.035 . The camber $y_{c}$, defined as the maximum displacement of the camber line from the chord line, is given here by $y_{c}=$ $-(1 / 16)\left(\Delta_{u}+\Delta_{\ell}\right) \epsilon^{2} / d$. In Fig. 3a, $y_{c}=0$; in Figs. 3b and 3c, $y_{c}=1 / 4 t / c$. For all cases the first term in the angle of attack is $\epsilon^{2} \alpha_{2}=\epsilon^{2} f_{u}(d) / d=-1 / 2 \epsilon^{2} \Delta_{u}(1-d) / d$. In Figs. 3a and $3 \mathrm{~b}$, the second term $\epsilon^{5 / 2} \alpha_{5 / 2}$ is chosen so that $m_{2}=0$ and there is no flow around the leading edges. In Fig. 3a, for symmetric blades, the Mach number $M_{0}$ is chosen such that the "channel" part of the solution describes a flow which is very nearly choked. This value, $M_{0}=0.815$, is also used in Figs. $3 \mathrm{~b}$ and $3 \mathrm{c}$. The maximum local Mach number, however, is decreased in Fig. 3b because of the added camber. In Fig. 3c, $\alpha_{5 / 2}$ is chosen so that $m_{2}<0$, giving a downward flow around the leading edge and an increased flow between the blades, again approaching the choked condition.

In each of the cases shown in Fig. 3, the flow accelerates over the front portion of a blade, and between the blades, in a region containing the minimum "channel" cross-sectional 


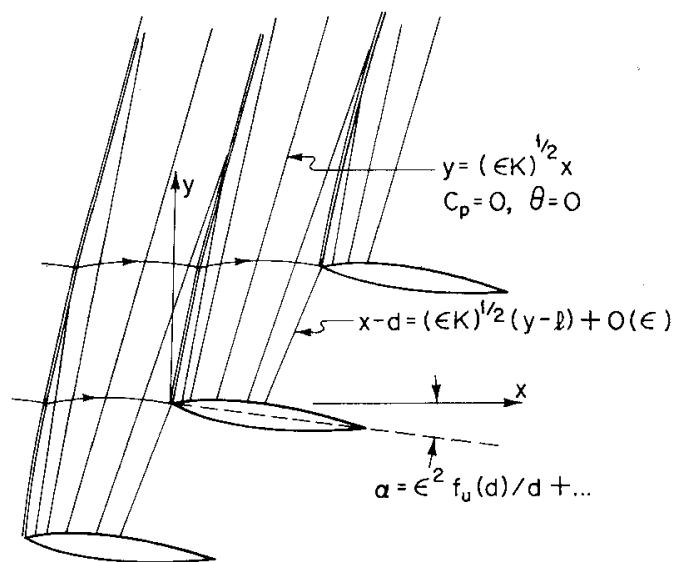

Fig. 5 Flow structure for cascade at low supersonic Mach number.

area, the flow resembles a channel flow. The minimum area occurs at $x \approx 0.78$ in Fig. $3 \mathrm{a}$ and at $x \approx 0.64$ in the remaining cases. "Choking" occurs if $\phi_{l}^{\prime}(x)$, found from Eq. (11) and negative for subsonic flow, increases to zero at this value of $x$; this one-dimensional condition was suggested for an unstaggered cascade in Ref. 16. The addition of camber in Fig. $3 \mathrm{~b}$ removes the near symmetry of the "channel" flow in Fig. 3a; the Mach number is decreased in the flow between the blades and also somewhat upstream. In the last case the decrease in angle of attack leads to an increase in mass flow and therefore an increase in local Mach number throughout most of the flow. In Figs. $3 a$ and $3 b$ the velocity pert arbation has a logarithmic singularity at the leading-edge siagnation point; in Fig. 3c the inverse-square-root singularity associated with flow around the edge is superimposed. All of the curves shown in Fig. 3 represent second-order solutions. In Fig. 4, results are shown for both the first and second approximations to the pressure coefficient at an upper airfoil surface for the same three sets of parameters. The secondorder correction is seen to be largest in the edge regions, and in fact seems to be a little too large near $x=d$, since a small bump appears in each of the second-order curves near that point. This irregularity appears to be primarily associated with the manner in which the near-field, leading-edge, and channel solutions were joined near the leading edge. ${ }^{12}$ There are many possible ways to accomplish this joining, and it should be possible to identify a rational procedure, consistent with the goal of achieving correct asymptotic representations, which leads to a smoothed form for the pressure distributions. This refinement is still under study.

Finally, the flow properties can also be calculated at large distances downstream. The flow direction is found by equating the mass flow between two adjacent airfoils, evaluated just upstream from the end of the "channel" region, and the mass flow between the same two streamlines far behind the cascade. The result for the streamline inclination angle $\theta_{f}$ far downstream, measured positive counterclockwise from the $x$ axis, is

$$
\theta_{f}=-\epsilon^{2} \frac{f_{u}(d)+f_{\ell}(l-d)}{d}-\epsilon^{5 / 2} \frac{K_{l} K_{3 / 2} \ell}{(\gamma+l) d}+\cdots
$$

where $K_{1}$ and $K_{3 / 2}$ are coefficients in the expansion of the Mach number $M_{f}$ far downstream:

$$
M_{f}^{2}=I-K_{l} \epsilon-K_{3 / 2}(\epsilon) \epsilon^{3 / 2}+\cdots
$$

Matching of channel, trailing-edge, and downstream outer solutions gives

$$
K_{I}=\left\{K^{2}+2(\gamma+1) \frac{f_{u}(d)+f_{\ell}(l-d)}{\ell}\right\}^{1 / 2}
$$

$$
\frac{K_{I}^{1 / 2} K_{3 / 2}(\epsilon)}{\gamma+1}=-\frac{\Delta_{l}}{\pi}+\frac{\alpha_{5 / 2} d}{K_{l}^{1 / 2} \ell}+\frac{\Delta_{\ell}}{\pi} \ln \frac{2\left(\epsilon K_{I}\right)^{1 / 2} \ell}{d}-\frac{2 \pi}{d} \sum_{n=1}^{\infty} n a_{n \ell}
$$

where $\Delta_{l}=f_{u}^{\prime}(1)-f_{\ell}^{\prime}(1-d), \quad \Delta_{\ell}=f_{p}^{\prime}(1)-f_{\ell}^{\prime}(1-d)$; terms of order $\epsilon^{3 / 2}$ and $\epsilon^{3 / 2} \ln \epsilon$ have been grouped together for convenience. The coefficients $a_{n \ell}$ are Fourier coefficients in a series analogous to Eq. (18), obtained for the lower surface for $1-d<x<1$. In the special case of a circular-arc airfoil, $f_{u}(d)+f_{\ell}(1-d)=-1 / 2\left(\Delta_{u}+\Delta_{\ell}\right)(1-d)$. The corresponding downstream value of the pressure can be found from the result for $M_{f}$. Thus the analytical solutions lead to relatively simple approximate expressions for the overall flow changes in terms of the various parameters.

\section{Supersonic Flows}

At low supersonic speeds in the range $M_{0}^{2}-1=O(\epsilon)$, the flow ahead of the cascade has a wave structure as sketched in Fig. 5. The airfoil leading edges are assumed sharp, so that the shock waves are necessarily attached, because the flow deflection angle of order $\epsilon^{2}$ is small in comparison with the maximum possible turning angle when $M_{0}^{2}-1=O(\epsilon)$. The characteristics are very nearly parallel to the shock waves, intersecting the shocks only at large distances, and so the shock-wave strength decreases slowly as $y$ increases. The flow between two successive shock waves is nearly a simple wave and flow properties are nearly constant along a characteristic. For each airfoil a limiting characteristic separates the disturbances which overtake the forward shock from those which are overtaken by the shock wave to the rear. At this characteristic the flow properties are the same as in the undisturbed stream. In a first approximation the direction of the undisturbed flow is then the direction of a tangent to the airfoil surface at the intersection with the limiting characteristic. This is the direction of the straight line $y=\epsilon^{2}\left\{x f_{u}(d) / d-\alpha_{2} x\right\}=0$; i.e., the average value of $f_{u}^{\prime}(x)-$ $\alpha_{2}$ for $0<x<d$ is zero. Outward wave propagation from the cascade therefore influences the flow at large distances upstream and implies a "unique incidence angle" equal, in a first approximation, to $\epsilon^{2} f_{u}(d) / d$. $^{1,13,14}$

Although each fluid element crosses infinitely many shock waves, the entropy changes and the vorticity are small enough to permit representation of the flow by the first few terms of a velocity potential expanded in the form of Eq. (20); it is shown later that the error is $O\left(\epsilon^{7 / 2}\right)$. Coordinates $\tilde{x}$ and $\tilde{y}$ are again defined by Eq. (19), but with the upper sign used in the definition of $K$ [Eq. (1)]. The term $\epsilon^{3 / 2} \tilde{\phi}_{3 / 2}$ describes a linearized flow past the scalloped wall shape sketched in Fig. $2 a$ and is found as a solution to the wave equation in the region between successive shock waves:

$$
\tilde{\phi}_{3 / 2}(\tilde{x} \tilde{y})=-K^{-1 / 2} f_{u}(\tilde{x}-\tilde{y})+(\tilde{x}-\tilde{y}) K^{-1 / 2} \alpha_{2}
$$

At a shock wave defined by

$$
S(x, y)=x-x_{s}(y)=0
$$

the mass flow $\rho q \cdot \nabla S /|\nabla S|$ and the potential $\phi$ are continuous. If a jump is denoted by square brackets, these two conditions become, respectively,

$$
\begin{gathered}
{\left[\rho\left(u_{0}+\phi_{x}-\phi_{y} x_{s}^{\prime}\right)\right]=0} \\
{\left[\phi_{y}+x_{s}^{\prime} \phi_{x}\right]=0}
\end{gathered}
$$

where $\rho$ is the nondimensional density. Evaluation of the first relation requires the expansion

$$
\rho u=1+k \epsilon-K \epsilon \phi_{x}-1 / 2(\gamma+l) \phi_{x}^{2}+\cdots
$$

The solution of Eq. (36) for $\tilde{\phi}_{3 / 2}$ is easily shown to be consistent with the largest terms in the two shock-wave jump conditions. 
Since the variation in slope of the exact characteristics is $O(\epsilon)$, the displacement from the linearized positions is $O(1)$ when $\tilde{y}=O\left(\epsilon^{-I}\right)$, i.e., when $\tilde{y}=O\left(\epsilon^{-1 / 2}\right)$. At these distances the solution in terms of $\tilde{x}$ and $\tilde{y}$ must be replaced by a far-field solution, obtained by an extension of the derivation for a single airfoil. ${ }^{15,17}$ Convenient variables are

$$
\hat{x}=\tilde{x}-\tilde{y} \quad \hat{y}=\epsilon^{1 / 2} \tilde{y}
$$

The potential has the form

$$
\phi=\epsilon^{3 / 2} \hat{\phi}_{3 / 2}(\hat{x}, \hat{y})+\epsilon^{2} \hat{\phi}_{2}(\hat{x}, \hat{y})+\cdots
$$

Combining the shock-wave relations [Eqs. (38) and (39)] leads, in the first approximation, to the familiar results that each shock wave bisects the angle between the characteristics immediately upstream and downstream and that the Riemann invariant for any incoming characteristic remains unchanged across a shock wave. Substitution of the expansion of Eq. (42) in the potential equation (6) leads to a first-order differential equation for $\hat{\phi}_{(3 / 2) \hat{x}}$ which states that $\hat{\phi}_{(3 / 2) \hat{x}}$ is constant along characteristics $\mathrm{d} \hat{x} / \mathrm{d} \hat{y}=K^{1 / 2} \lambda+1 / 2(\gamma+I) K^{-1} \hat{\phi}_{(3 / 2) \hat{x}}$. The solution which satisfies the proper matching condition as $\hat{y} \rightarrow 0$ is

$$
\hat{\phi}_{(3 / 2) \hat{x}}(\hat{x}, \hat{y})=K^{-1 / 2}\left\{-f_{u}^{\prime}(X)+\alpha_{2}\right\}
$$

where $X=$ const along a characteristic and is defined in terms of $\hat{x}$ and $\hat{y}$ by

$$
X-\hat{x}+\hat{y}\left\{K^{1 / 2} \lambda-1 / 2(\gamma+1) K^{-3 / 2}\left[f_{u}^{\prime}(X)-\alpha_{2}\right]\right\}=0
$$

so that $x=\hat{x}=X$ at the intersection of the characteristic and the $x$ axis. Thus in a first approximation $u=$ const along characteristics $X=$ const. An expression for $\hat{\phi}_{(3 / 2) \hat{y}}$, needed if a solution for $\hat{\phi}_{2}$ is also to be derived, is found by combining the two shock-wave relations for $\hat{\phi}_{3 / 2}$ with a first integral of the differential equation for $\hat{\phi}_{3 / 2}$, noting also that no disturbances originate upstream. The result is

$$
\hat{\phi}_{(3 / 2) \hat{y}}+K^{1 / 2} \lambda \hat{\phi}_{(3 / 2) \hat{x}}+\frac{\gamma+1}{4 K} \hat{\phi}_{(3 / 2) \hat{x}}^{2}=0
$$

The shock waves are represented by

$$
\hat{x}-n d=\hat{x}_{s}(\hat{y} ; \epsilon)=\hat{x}_{s 0}(\hat{y})+\cdots
$$

for $n=0, \pm 1, \pm 2, \ldots$. Since the shock-wave slope, in a first approximation, is the average of the slopes of characteristics immediately upstream and downstream,

$$
\frac{\mathrm{d} \hat{x}_{s 0}}{\mathrm{~d} \hat{y}}=K^{1 / 2} \lambda-\frac{\gamma+1}{4 K^{3 / 2}}\left\{f_{u}^{\prime}\left(X_{+}\right)+f_{u}^{\prime}\left(X_{-}\right)-2 \alpha_{2}\right\}
$$

where the values $X_{-}$and $X_{+}$identify, respectively, the upstream and downstream characteristics which intersect the shock wave at any given point. A pair of differential equations for $X_{-}$and $X_{+}$as functions of $\hat{y}$ is obtained if Eq. (44) for the characteristic curves is evaluated just ahead of and behind a shock wave, differentiated with respect to $\hat{y}$, and then combined with Eq. (47):

$$
\begin{aligned}
& \left(2 \hat{y} \frac{\mathrm{d} \theta_{-}}{\mathrm{d} X_{-}}-\frac{4 K^{3 / 2}}{\gamma+1}\right) \frac{\mathrm{d} X_{-}}{\mathrm{d} \hat{y}}=\theta_{+}-\theta_{-} \\
& \left(2 \hat{y} \frac{\mathrm{d} \theta_{+}}{\mathrm{d} X_{+}}-\frac{4 K^{3 / 2}}{\gamma+1}\right) \frac{\mathrm{d} X_{+}}{\mathrm{d} \hat{y}}=-\theta_{+}+\theta_{-}
\end{aligned}
$$

where $\theta_{-}=f_{u}^{\prime}\left(X_{-}\right)-\alpha_{2}$ and $\theta_{+}=f_{u}^{\prime}\left(X_{+}\right)-\alpha_{2}$. For a given airfoil surface $y=\epsilon^{2}\left\{f_{u}(x)-\alpha_{2} x\right\}, \quad 0<x<d, \quad$ numerical integration of Eqs. (47-49) allows calculation of the shockwave shape. By subtracting Eqs. (48) and (49), or by evaluating Eq. (44) at $X=X_{-}$and at $X=X_{+}$and subtracting, one finds also

$$
2 \hat{y}\left(\theta_{+}-\theta_{-}\right)=\frac{4 K^{3 / 2}}{\gamma+1}\left(X_{+}-X_{-}\right)
$$

Since $X_{+}-X_{-} \rightarrow d$ as $\hat{y} \rightarrow \infty$, the shock-wave strength as measured by $\theta_{+}-\theta_{-}$is proportional to $1 / \hat{y}$ as $\hat{y} \rightarrow \infty$. This dependence on distance is equivalent to the time rate of decay derived from nonlinear acoustics for one-dimensional propagation of a sawtooth waveform. ${ }^{18}$

Another useful form is the expression for $\mathrm{d} X_{+} / \mathrm{d} X$ found by dividing Eqs. (48) and (49). In particular, for a circular-arc airfoil $\mathrm{d} X_{+} / \mathrm{d} X_{-}=-1$ and therefore $X_{+}=-X_{-}$. If the shock wave in the original variables is given by $x=x_{s}(y ; \epsilon)$, the slope becomes $\mathrm{d} x_{s} / \mathrm{d} y=(K \epsilon)^{1 / 2}+o(\epsilon)$. That is, because of the special geometry the shock waves remain straight for all $y$, at least to this order of approximation. The corresponding pressure coefficient, if $f_{u}(x)=2 x(1-x)$, is found ${ }^{12}$ to be

$$
c_{p} \sim-\frac{8 \epsilon^{3 / 2}}{K^{1 / 2}} \frac{x-1 / 2 d-(\epsilon K)^{1 / 2}(y-\lambda x)-\epsilon K \lambda(y-\lambda x)}{I+2(\gamma+1) K^{-1} \epsilon(y-\lambda x)}
$$

for $y>0$ and $0<x-(K \epsilon)^{1 / 2} y+\ldots<d-(\epsilon K)^{1 / 2} \ell+\ldots$. Thus for a circular-arc profile the pressure coefficient in the region between two successive shock waves is a linear function of $x$ at any prescribed value of $y-\lambda x$, with slope which decreases in magnitude as $y$ increases. The result of Eq. (51) is uniformly valid to order $\epsilon^{3 / 2}$ for all $y>0$.

These far-field solutions are consistent with the assumption that the vorticity is of sufficiently high order to be neglected here. The changes in pressure and entropy across a shock wave are $O\left(\epsilon^{3 / 2}\right)$ and $O\left(\epsilon^{9 / 2}\right)$, respectively, and are functions of $\hat{y}$; as $\hat{y} \rightarrow \infty$ the changes are $O\left(\epsilon^{3 / 2} / \hat{y}\right)$ and $O\left(\epsilon^{9 / 2} / \hat{y}^{3}\right)$. Each fluid element, however, crosses an infinite number of shock waves. Since the flow is periodic, the overall entropy increase can instead be written as a summation of changes at points along a single shock wave. The distance between successive points is $\hat{y}_{n}-\hat{y}_{n-I}=K^{1 / 2} \in$. As $\epsilon \rightarrow 0$, the sum approaches the product of $\left(K^{1 / 2} \ell\right)^{-1}$ and the integral of the entropy jump. The overall entropy increase is therefore $O\left(\epsilon^{7 / 2}\right)$. The vorticity is proportional to the entropy gradient and thus has magnitude $O\left(\epsilon^{9 / 2}\right)$, so that the first term in the velocity component $u$ which cannot be found from a potential is $O\left(\epsilon^{7 / 2}\right)$.

Higher approximations can also be derived. The second term in the expansion of $\phi$ for $\tilde{y}=O(1)$ can be expressed by the sum $\tilde{\phi}_{2}=\tilde{\phi}_{2}^{(p)}+\tilde{\phi}_{(}^{(h)}$, where $\tilde{\phi}_{2}$ satisfies a nonhomogeneous wave equation, $\tilde{\phi}_{2}^{(p)}$ is a particular solution, and $\tilde{\phi}_{2}^{(h)}$ satisfies the homogeneous equation. Here $\tilde{\phi}_{2}^{(p)}$ can be taken to have the form of Eq. (30), but with the signs of both terms changed. It follows from expanding and combining the two shock-wave relations that $\phi_{2}^{(p)}$ then satisfies the proper jump condition for the quantity $\tilde{\phi}_{2 \bar{x}}+\tilde{\phi}_{2 \bar{y}}$, so that $\tilde{\phi}_{2}^{(h)}$ does not contribute to this jump. Since also no incoming disturbances are present at large distances, $\tilde{\phi}_{2 \bar{x}}^{(h)}$ and $\tilde{\phi}_{2 \bar{y}}^{(h)}$ are functions only of $\tilde{x}-\tilde{y}$. The complete solution for $\phi_{2}$ is

$$
\begin{aligned}
& \tilde{\phi}_{2}(\bar{x}, \tilde{y})=\tilde{y}\left\{\lambda f_{u}^{\prime}(\tilde{x}-\tilde{y})+\frac{\gamma+1}{2 K^{2}} \alpha_{2} f_{u}^{\prime}(\tilde{x}-\tilde{y})\right. \\
& \left.\quad-\frac{\gamma+1}{4 K^{2}} f_{u}^{\prime 2}(\tilde{x}-\tilde{y})\right\}+C \tilde{x}+(\tilde{x}-\tilde{y})\left\{K^{-1 / 2} \alpha_{5 / 2}\right. \\
& \left.\quad+\lambda f_{u}^{\prime}(\tilde{x}-\tilde{y})\right\}+\frac{\gamma+1}{4 K^{2}}\left\{2 \alpha_{2} f_{u}(\tilde{x}-\tilde{y})-\int_{0}^{\tilde{x}-\tilde{y}} f^{\prime 2}(t) \mathrm{d} t\right\}
\end{aligned}
$$


where the constants $\alpha_{5 / 2}$ and $C$ are as yet unknown. Since the streamline displacement is periodic, the integral of $\tilde{\phi}_{2 \bar{y}}$ is zero along a line $\tilde{y}=$ const between two successive shock waves. Matching the expansion of $\phi_{y}$ as $\tilde{y} \rightarrow 0$ with the simple solution for $y=O(1)$, given by Eq. (16) with $-K$ replaced by $K$, leads to the value $\alpha_{5 / 2}=\left(K^{1 / 2} \lambda / d\right)\left\{f_{u}(d)-d f_{u}^{\prime}(d)\right\}$. The second approximation to the unique incidence angle, expanded in the form $\alpha=\epsilon^{2} \alpha_{2}+\epsilon^{5 / 2} \alpha_{5 / 2}+\ldots$, is then given by

$$
\alpha=\epsilon^{2} f_{u}(d) / d+\epsilon^{5 / 2}\left(K^{1 / 2} \lambda / d\right)\left\{f_{u}(d)-d f_{u}^{\prime}(d)\right\}+\cdots
$$

For this angle of attack the upper-surface ordinate $y=\epsilon^{2} f_{u}(x)-\alpha x$ is zero, to order $\epsilon^{5 / 2}$, at the intersection of the airfoil surface and the last Mach line $x-d \sim(\epsilon K)^{1 / 2}(y-\ell)$ which passes ahead of the leading edge of the next airfoil (Fig. 5). ${ }^{1,13,14}$ That is, Eq. (53) is the result that would be obtained by expansion of $\epsilon^{2} \alpha_{2}$ if $d$ were replaced by $d-(K \epsilon)^{1 / 2} \ell$. Since $\phi$ is continuous across shock waves and since the flow is periodic in $\tilde{x}$, the value of $\phi$ at a given $\tilde{y}$ just behind one shock equals the value of $\phi$ at the same $\tilde{y}$ just ahead of the next shock. If the solution $\epsilon^{3 / 2} \tilde{\phi}_{3 / 2}+\epsilon^{2} \tilde{\phi}_{2}+\ldots$ is expanded about the positions of two successive shock waves, it is found that the terms proportional to $\tilde{y}$ cancel, and the value of $C$ is

$$
C=-\frac{\lambda f_{u}(d)}{d}-\frac{\gamma+1}{2 K^{2}} \frac{f_{u}^{2}(d)}{d^{2}}+\frac{\gamma+1}{4 K^{2} d} \int_{0}^{d} f^{\prime 2}(t) \mathrm{d} t
$$

Expansion of $\tilde{\phi}_{2 \tilde{x}}$ as $\tilde{y} \rightarrow 0$ now permits matching with the solution of Eq. (15) obtained for $y=O(1)$, and the function $h_{2}(x)$ in Eq. (15) is thereby determined. Terms of order $\epsilon^{3 / 2}$ and $\epsilon^{2}$ in the pressure coefficient at the airfoil surface, for $0<x<d$, can then be calculated from

$$
c_{p}=-2\left(\frac{u}{u_{0}}-1\right)+\cdots
$$

Finally, a second approximation for the far-field, if desired, could be calculated by a straightforward extension of the procedure of Ref. 15. Expansion of $\tilde{\phi}_{2 \tilde{x}}$ as $\tilde{y} \rightarrow \infty$ would provide the terms needed for matching with the far-field solution.

\section{Concluding Remarks}

For the class of transonic cascade flows considered, analytical results have been derived for the velocity distributions throughout the flow in the subsonic case and everywhere ahead of the airfoils in the supersonic case. Overall changes in Mach number and flow direction are found explicitly in terms of the parameters for the subsonic case. For a given geometry, if the normal distance between airfoils has a minimum between $x=d$ and $x=1$, no solution is obtained for a range of Mach numbers including $M_{0}=1$ because of massflow limitations. A few example numerical results show realistic trends, but no attempt has yet been made at a thorough numerical study. Before this is done, it would be of interest to study other limiting cases which would allow more heavily loaded airfoils; moreover, some improvements are needed in the numerical procedure used for the evaluation of the analytical solutions. ${ }^{12}$ As the next step, however, it seems most desirable to study an appropriate limiting case for threedimensional compressor flow, so as to understand in detail the manner in which parts of the three-dimensional problem may reduce to two-dimensional problems of the type considered here. Such a study is in progress.

Aside from providing results which can be valuable in their own right, the analytical solutions have a number of other potential applications. The pressure and Mach number distributions allow at least a good first guess for use in numerical solutions for flows through lightly loaded cascades having blade spacing comparable to the chord. The predicted distributions also help to identify the regions where large rates of change are to be expected, and analytical far-field solutions might be of use in the formulation of boundary conditions at large distances. The explicit dependence on parameters shown in the analytical solutions can perhaps allow extrapolation of experimental results to other cases, or may suggest procedures for correlation of experimental data. This might be accomplished through the use of similarity laws based on the expressions derived for the overall flow changes. Finally, as already noted, asymptotic formulations are also possible for two-dimensional flows corresponding to other parameter ranges and for three-dimensional flows. Additional analytical solutions will contribute to improved understanding of flow details. One of the ultimate goals, of course, is to provide information for direct use in predictions of rotor performance.

\section{Acknowledgments}

This work was supported in part by the Office of Naval Research; this support is gratefully acknowledged. The authors also wish to express their thanks to Mr. S. Agrawal for carrying out the numerical calculations.

\section{References}

${ }^{1}$ Hawthorne, W. R. (Ed.), Aerodynamics of Turbines and Compressors, Princeton University Press, Princeton, N. J., 1964.

${ }^{2}$ Starken, H. and Lichtfuss, H. J., "Supersonic Cascade Performance,"'Advanced Compressors, AGARD-LS-39-70, 1970.

${ }^{3}$ Adamson, T. C. Jr. and Platzer, M. F. (Eds)., Transonic Flow Problems in Turbomachinery, Hemisphere Publishing Corp., Washington, D. C., 1977.

${ }^{4}$ Cole, J. D., "Modern Developments in Transonic Flow," SIAM Journal on Applied Mathematics, Vol. 29, Dec. 1975, pp. 763-787.

${ }^{5}$ Hayes, W. D., "La Seconde Approximation pour les Ecoulements Transsoniques non Visqueux," Journal de Mecanique, Vol. 5, June 1966, pp. 163-206.

${ }^{6}$ Szaniawski, A., "Transonic Approximations to the Flow through a Nozzle," Archiwum Mechaniki Stosowanej, Vol. 17, 1965, pp. 7985.

${ }^{7}$ Adamson, T. C. Jr., Messiter, A. F., and Richey, G. K., "On the Matching of Solutions for Unsteady Transonic Nozzle Flows," Archiwum Mechaniki Stosowanej, Vol. 26, 1974, pp. 617-628.

${ }^{8}$ Messiter, A. F. and Adamson, T. C. Jr., "On the Flow near a Weak Shock Wave Downstream of a Nozzle Throat," Journal of Fluid Mechanics, Vol. 69, 1975, pp. 97-108.

${ }^{9}$ Richey, G. K. and Adamson, T. C. Jr., "Analysis of Unsteady Transonic Channel Flow with Shock Waves," AIAA Journal, Vol. 14, Aug. 1976, pp. 1054-1061.

${ }^{10}$ Chan, J., S. K. and Adamson, T. C. Jr., "Unsteady Transonic Flows with Shock Waves in an Asymmetric Channel," $A I A A$ Journal, Vol. 16, April 1978, pp. 377-384.

${ }^{11}$ Adamson, T. C. Jr., Messiter, A. F., and Liou, M. S., "Large Amplitude Shock-Wave Motion in Two-Dimensional, Transonic Channel Flows," AIAA Journal, Vol. 16, Dec. 1978, pp. 1240-1247.

${ }^{12}$ Messiter, A. F. and Adamson, T. C. Jr., "Transonic SmallDisturbance Theory for Lightly Loaded Cascades," University of Michigan Rept. N00014-79-C-0285-T2, Feb. 1981.

${ }^{13}$ Kantrowitz, A., "The Supersonic Axial-Flow Compressor," NACA Rept. 974, 1950.

${ }^{14}$ Kurosaka, M., "On the Unsteady Supersonic Cascade with a Subsonic Leading Edge, Part I: An Exact First Order Theory," Transactions of ASME, Journal of Engineering for Power, Vol. 96, Series A, Jan. 1974, pp. 13-22.

${ }^{15}$ Caughey, D. A., "Second-Order Wave Structure in Supersonic Flows," NASA CR-1438, Sept. 1969.

${ }^{16}$ Ackeret, J. and Rott, N., "Uber die Strömung von Gasen durch ungestaffelte Profilgitter," Schweizerische Bauzeitung, Vol. 67, Jan. 1949, pp. 40-41, 58-61.

${ }^{17}$ Lighthill, M. J., "'Higher Approximations,"' General Theory of High Speed Aerodynamics, edited by W. R. Sears, Princeton University Press, Princeton, N. J., 1954, pp. 345-489.

${ }^{18}$ Whitham, G. B., Linear and Nonlinear Waves, John Wiley and Sons, New York, 1974. 